\title{
Interaction of laminar far wake with a free surface
}

\author{
Andy T. Chan and Allen T. Chwang ${ }^{\text {a) }}$ \\ Department of Mechanical Engineering, The University of Hong Kong, Pokfulam Road, Hong Kong
}

(Received 15 February 1995; accepted 30 October 1995)

\begin{abstract}
Wave disturbances caused by the uniform translatory motion of a submerged body on or beneath the free surface of a viscous fluid are investigated analytically. The submerged body is idealized as an Oseenlet or an Oseen doublet, and exact solutions in closed integral forms are obtained. Based on these exact solutions, asymptotic representations of the wave amplitude for large Reynolds numbers based on the deep-water wavelength at large distances downstream of the body are derived. The results obtained show explicitly the effect of the laminar wake on the amplitude and the phase of the surface waves thus created. (C) 1996 American Institute of Physics. [S1070-6631(96)02902-X]
\end{abstract}

\section{INTRODUCTION}

A submerged body moving through an incompressible viscous fluid generates waves on the free surface of the fluid. Generation of free-surface waves due to a moving disturbance can be found in many situations, such as a ship moving across the ocean or a fish swimming near the free surface. In the present study, we investigate the waves generated by bodies with uniform translatory motion near the free surface of a viscous fluid. We simulate the moving body by an Oseenlet or an Oseen doublet, which creates a laminar far wake downstream of itself similar to that due to the moving body.

A great deal of work has been devoted to problems of this kind, since Kelvin's studies ${ }^{1}$ of ship waves. Lamb ${ }^{2}$ and De Prima and $\mathrm{Wu}^{3}$ provided analytical solutions for various cases of the problem. Wehausen and Laitone $e^{4}$ first provided methodologies in understanding viscous waves using infinitesimal wave theory. Cumberbatch ${ }^{5}$ also worked on viscous waves employing similar methodologies for a pressure point. Dugan $^{6}$ dealt with a two-dimensional version of the problem by studying the viscous drag of bodies moving near a free surface, while Ammicht ${ }^{7}$ extended Dugan's work by considering the effect of surface tension. The corresponding threedimensional Green's tensor problem was formulated by Lurye. ${ }^{8}$ However, it was felt that the effect of the laminar far wake has not been fully explored, which in this paper is shown to be of significant interest.

Our analysis is based on the assumptions that the motion satisfies the Oseen's equations, the fluid is infinitely deep, and the free-surface conditions are linearized. We shall derive formal expressions for the wave amplitude associated with the laminar wake created by the motion of an Oseenlet or an Oseen doublet parallel or perpendicular to the free surface. Then the asymptotic behavior of these expressions is derived and graphical plots of the system shown.

\section{THEORETICAL SOLUTION FOR A HORIZONTAL OSEENLET}

We consider first a viscous incompressible fluid that occupies the lower half space $z<0$ in a Cartesian coordinate system and is flowing with a uniform velocity $U \mathbf{e}_{x}$, where $\mathbf{e}_{x}$ denotes the unit vector along the $x$ direction. Let us nondimensionalize the velocity by $U$, the pressure by $\rho U^{2}$, and distance by $U^{2} / g$, where $\rho$ is the density of the fluid and $g$ is the gravitational constant. For small disturbances, the perturbed dimensionless velocity $\mathbf{u}=(u, v, w)$ satisfies the steady-state Oseen's equations,

$$
\begin{aligned}
& \boldsymbol{\nabla} \cdot \mathbf{u}=0, \\
& \frac{\partial \mathbf{u}}{\partial x}=-\nabla p+\epsilon \nabla^{2} \mathbf{u},
\end{aligned}
$$

where

$$
\epsilon=\frac{\mu g}{\rho U^{3}},
$$

$p$ is the dimensionless pressure, and $\mu$ is the dynamic viscosity of the fluid. The dimensionless parameter $\epsilon$ in (3) can be regarded as the reciprocal of the Reynolds number with respect to the deep-water wavelength $U^{2} / g$. It will be used as the perturbation parameter throughout the asymptotic expansion of the free-surface elevation expression.

For a singular force (Oseenlet) located at $\left(0,0,-z_{0}\right)$ with dimensionless magnitude $4 \pi F$, normalized with respect to $\rho U^{2}\left(U^{2} / g\right)^{2}$, along the negative $x$ direction, the solution to Eqs. (1) and (2) for an unbounded fluid is given by Moore ${ }^{9}$ as

$$
\begin{aligned}
& \mathbf{u}=-\frac{F}{\epsilon} \frac{e^{(x-r) / 2 \epsilon}}{r} \mathbf{e}_{x}+F \boldsymbol{\nabla}\left(\frac{e^{(x-r) / 2 \epsilon}-1}{r}\right), \\
& p=-\frac{F x}{r^{3}},
\end{aligned}
$$

where $\mathbf{e}_{x}$ denotes the unit vector in the $x$ direction and

$$
r^{2}=x^{2}+y^{2}+\left(z+z_{0}\right)^{2} .
$$

We impose the linearized free surface conditions at $z=0$ that represent the continuity of the normal and tangential stresses in addition to the kinematic boundary condition, ${ }^{4}$

$$
\begin{aligned}
& \frac{\partial u}{\partial z}+\frac{\partial w}{\partial x}=0, \\
& \frac{\partial v}{\partial z}+\frac{\partial w}{\partial y}=0,
\end{aligned}
$$

\footnotetext{
e-mail: atchwang@hkucc.hku.hk
} 


$$
\frac{\partial p}{\partial x}-2 \epsilon \frac{\partial^{2} w}{\partial x \partial z}-w=0 .
$$

Now let the entire solution be written as

$$
\begin{aligned}
& \mathbf{u}=\mathbf{u}_{u}+\mathbf{u}_{\mathrm{FS}}, \\
& p=p_{u}+p_{\mathrm{FS}},
\end{aligned}
$$

where subscript $u$ denotes the unbounded system solution given by (4) and (5) and FS denotes the free-surface effect. In terms of $\mathbf{u}_{\mathrm{FS}}$, the free-surface conditions become

$$
\begin{aligned}
& \frac{\partial u_{\mathrm{FS}}}{\partial z}+\frac{\partial w_{\mathrm{FS}}}{\partial x}=-\left(\frac{\partial u_{u}}{\partial z}+\frac{\partial w_{u}}{\partial x}\right), \\
& \frac{\partial v_{\mathrm{FS}}}{\partial z}+\frac{\partial w_{\mathrm{FS}}}{\partial y}=-\left(\frac{\partial v_{u}}{\partial z}+\frac{\partial w_{u}}{\partial y}\right), \\
& \frac{\partial p_{\mathrm{FS}}}{\partial x}-2 \epsilon \frac{\partial^{2} w_{\mathrm{FS}}}{\partial x \partial z}-w_{\mathrm{FS}}=-\left(\frac{\partial p_{u}}{\partial x}-2 \epsilon \frac{\partial^{2} w_{u}}{\partial x \partial z}-w_{u}\right) .
\end{aligned}
$$

To reduce the number of variables involved, we define two new functions $\phi$ and $\mathbf{f}$ by

$$
\begin{aligned}
& \mathbf{u}_{\mathrm{FS}}=-\boldsymbol{\nabla} \phi+\boldsymbol{\nabla} \times \mathbf{f}, \\
& \mathbf{f}=f_{x} \mathbf{e}_{x}+f_{z} \mathbf{e}_{z}, \\
& p_{\mathrm{FS}}=\frac{\partial \phi}{\partial x},
\end{aligned}
$$

such that

$$
\begin{aligned}
& \nabla^{2} \phi=0, \\
& \frac{\partial \mathbf{f}}{\partial x}=\epsilon \nabla^{2} \mathbf{f},
\end{aligned}
$$

for $z<0$. Thus, we can express the linearized free-surface conditions in terms of $\phi$ and f. Combining Eqs. (12)-(19), we obtain, at $z=0$,

$$
\begin{aligned}
& -2 \frac{\partial^{2} \phi}{\partial x \partial z}+\frac{\partial^{2} f_{z}}{\partial y \partial z}-\frac{\partial^{2} f_{x}}{\partial x \partial y}=-\left(\frac{\partial u_{u}}{\partial z}+\frac{\partial w_{u}}{\partial x}\right), \\
& -2 \frac{\partial^{2} \phi}{\partial y \partial z}-\frac{\partial^{2} f_{z}}{\partial x \partial z}-\frac{\partial^{2} f_{x}}{\partial y^{2}}+\frac{\partial^{2} f_{x}}{\partial z^{2}}=-\left(\frac{\partial v_{u}}{\partial z}+\frac{\partial w_{u}}{\partial y}\right), \\
& \frac{\partial^{2} \phi}{\partial x^{2}}-2 \epsilon\left(\frac{\partial^{3} \phi}{\partial x \partial z^{2}}+\frac{\partial^{3} f_{x}}{\partial x \partial y \partial z}\right)+\left(\frac{\partial \phi}{\partial z}+\frac{\partial f_{x}}{\partial y}\right) \\
& =-\left(\frac{\partial p_{u}}{\partial x}-2 \epsilon \frac{\partial^{2} w_{u}}{\partial x \partial z}-w_{u}\right) .
\end{aligned}
$$

To solve the problem, it is convenient to define for $z \leqslant 0$ the following Fourier transforms for $j=1$ or 3 ( 1 and 3 denote $x$ and $z$, respectively),

$$
\begin{aligned}
& \phi=F \int_{-\infty}^{\infty} \int_{-\infty}^{\infty} \hat{\phi}\left[k_{1}, k_{2}\right] e^{i\left(k_{1} x+k_{2} y\right)+A z} d k_{1} d k_{2}, \\
& f_{j}=F \int_{-\infty}^{\infty} \int_{-\infty}^{\infty} \hat{f}_{j}\left[k_{1}, k_{2}\right] e^{i\left(k_{1} x+k_{2} y\right)+B z} d k_{1} d k_{2},
\end{aligned}
$$

where

$$
A=\sqrt{k_{1}^{2}+k_{1}^{2}}, \quad B=\sqrt{k_{1}^{2}+k_{2}^{2}+\frac{i k_{1}}{\epsilon}} .
$$

It can be shown that the free-surface elevation $\eta$ can be expressed as

$$
\eta=\left.p\right|_{z=0}-\left.2 \epsilon \frac{\partial w}{\partial z}\right|_{z=0} .
$$

The wave amplitude expression can also be written as

$$
\eta=\eta_{u}+\eta_{\mathrm{FS}},
$$

where

$$
\eta_{\mathrm{FS}}=\left.p\right|_{z=0}-\left.2 \epsilon \frac{\partial w_{\mathrm{FS}}}{\partial z}\right|_{z=0} .
$$

Introducing the cylindrical coordinates $(R, \theta)$ on the free surface through

$$
x=R \cos \theta, \quad y=R \sin \theta,
$$

it can be shown that

$$
\lim _{R \rightarrow \infty} \eta_{u}=o\left[\eta_{\mathrm{FS}}\right] .
$$

Thus

$$
\eta \approx \eta_{\mathrm{FS}}=\left.\frac{\partial \phi}{\partial x}\right|_{z=0}-\left.2 \epsilon\left(\frac{\partial^{2} f_{x}}{\partial y \partial z}-\frac{\partial^{2} \phi}{\partial z^{2}}\right)\right|_{z=0} .
$$

We then decompose the functions $\hat{\phi}$ and $\hat{f}_{x}$ into

$$
\hat{\phi}=\hat{\phi}_{0}+\hat{\phi}_{v}, \quad \hat{f}_{x}=\hat{f}_{0}+\hat{f}_{v},
$$

where subscript 0 denotes contribution by the inviscid term $\boldsymbol{\nabla}[1 / r]$ of the Lagerstrom needle and the subscript $v$ denotes the remaining viscous terms. Combining (20)-(32), we obtain

$\hat{\phi}_{0}=\frac{\left[-\left(k_{1}^{2} / 2 A+\frac{1}{2}\right)+2 i \epsilon k_{1} A+2 \epsilon^{2} A^{2}(A+B)\right] e^{-A z_{0}}}{\Delta}$,

$\hat{\phi}_{v}=\frac{\left(-2 i \epsilon k_{1} B-4 \epsilon^{2} A^{3} B\right) e^{-B z_{0}}}{\Delta}$,

$\hat{\phi}_{0}=\frac{\left(-2 \epsilon k_{1} A^{2}+4 i \epsilon^{2} A^{4}\right) e^{-A z_{0}}}{k_{2} \Delta}$,

$\hat{f}_{v}=\frac{\left[(i / 2)\left(k_{1}^{2}-A\right)+2 \epsilon k_{1} A^{2}-2 i \epsilon^{2} A^{4}-2 i \epsilon^{2} A^{3} B\right] e^{-B z_{0}}}{k_{2} \Delta}$,

where

$$
\Delta=\left(k_{1}^{2}-A\right)-4 i \epsilon k_{1} A^{2}+4 \epsilon^{2} A^{3}(B-A) .
$$

Hence, the exact integral expressions of the wave amplitude can be written as

$$
\begin{aligned}
& \eta_{\mathrm{FS}}^{0}=F \int_{-\infty}^{\infty} \int_{-\infty}^{\infty} h_{0} e^{i R\left(k_{1} \cos \theta+k_{2} \sin \theta\right)} d k_{1} d k_{2}, \\
& \eta_{\mathrm{FS}}^{v}=F \int_{-\infty}^{\infty} \int_{-\infty}^{\infty} h_{v} e^{i R\left(k_{1} \cos \theta+k_{2} \sin \theta\right)} d k_{1} d k_{2},
\end{aligned}
$$

where 


$$
\begin{aligned}
& h_{0}=\left(i k_{1}+2 \epsilon A^{2}\right) \hat{\phi}_{0}+2 i \epsilon k_{2} B \hat{f}_{0}, \\
& h_{v}=\left(i k_{1}+2 \epsilon A^{2}\right) \hat{\phi}_{v}+2 i \epsilon k_{2} B \hat{f}_{v} .
\end{aligned}
$$

To obtain the leading term in the expansion of the asymptotic representation for large $R$ of $\eta_{\mathrm{FS}}$, we shall employ Lighthill's two-stage scheme. ${ }^{10}$ We first consider the roots of the pole equation,

$$
\Delta\left[k_{1}, k_{2}, \epsilon\right]=\left(k_{1}^{2}-A\right)-4 i \epsilon k_{1} A^{2}+4 \epsilon^{2} A^{3}(B-A)=0 .
$$

For small $\epsilon$, Eq. (42) has two simple roots, which we shall call $k_{1,1}$ and $k_{1,2}$. From step 1 of Lighthill's scheme, we obtain from (38) and (39) for large $R$,

$$
\begin{aligned}
& \eta_{\mathrm{FS}}^{0}=i F \sum_{j=1}^{2} \int_{-\infty}^{\infty} \operatorname{Res}\left[h_{0}\right] e^{i R\left(k_{1, j} \cos \theta+k_{2} \sin \theta\right)} d k_{2}, \\
& \eta_{\mathrm{FS}}^{v}=i F \sum_{j=1}^{2} \int_{-\infty}^{\infty} \operatorname{Res}\left[h_{v}\right] e^{i R\left(k_{1, j} \cos \theta+k_{2} \sin \theta\right)} d k_{2},
\end{aligned}
$$

where $\operatorname{Res}[f]$ denotes the residue of function $f$.

In order to satisfy the pole equation, we introduce the following expansion of $k_{1}$ with respect to $\epsilon$. For small $\epsilon$, the two roots $k_{1, j}\left[k_{2}, \epsilon\right]$ take the form of

$$
\begin{aligned}
& k_{1, j}\left[k_{2}, \epsilon\right] \approx k_{1, j 0}\left[k_{2}\right]+i \epsilon k_{1, j 1}\left[k_{2}\right], \\
& k_{1, j 0}\left[k_{2}\right]=\frac{(-1)^{j}}{2} \sqrt{1+m\left[k_{2}\right]}, \\
& k_{1, j 1}\left[k_{2}\right]=\frac{\left(1+m\left[k_{2}\right]\right)^{3}}{2 m\left[k_{2}\right]},
\end{aligned}
$$

where $m\left[k_{2}\right]$ is a positive function defined by

$$
m\left[k_{2}\right]=\sqrt{1+4 k_{2}^{2}} .
$$

Since the residue factors $\operatorname{Res}\left[h_{0}\right]$ and $\operatorname{Res}\left[h_{v}\right]$ do not depend on $R$ but only on $\epsilon$ and $k_{2}$, it is convenient to replace them by their respective leading terms in their expansions about $\epsilon=0$, because only these terms contribute significantly to the asymptotic expressions of the wave amplitude. ${ }^{11}$ Thus

$$
\begin{aligned}
\operatorname{Res}\left[h_{0}\right]= & -i \frac{1+m\left[k_{2}\right]}{2 m\left[k_{2}\right]} e^{-\left[z_{0}\left(1+m\left[k_{2}\right]\right) / 2\right]}+O[\epsilon] \\
\operatorname{Res}\left[h_{v}\right]= & \epsilon^{1 / 2} \frac{2(-1)^{j-1}}{m\left[k_{2}\right]}\left(\frac{1+m\left[k_{2}\right]}{2}\right)^{7 / 4} \\
& \times e^{-\left(z_{0} / \sqrt{2 \epsilon}\left[\left(1+m\left[k_{2}\right]\right) / 2\right]^{1 / 4}\left[1+i(-1)^{j-1}\right]+i\left[(-1)^{j-1} \pi / 4\right]\right.} \\
& +o\left[\epsilon^{1 / 2} e^{\sqrt{i k_{1, j 0} / \epsilon}}\right]
\end{aligned}
$$

Examination of (49) and (50) shows that the wave amplitude can be expanded in the square root power of $\epsilon$, that is,

$$
\eta_{\mathrm{FS}}=\eta_{0}+\epsilon^{1 / 2} \eta_{1}+O[\epsilon],
$$

and $\eta_{\mathrm{FS}}^{0}$ contributes to the leading term $\eta_{0}$ while $\eta_{\mathrm{FS}}^{v}$ contributes to the term $\eta_{1}$.

The integral can now be evaluated by the steepestdescent method. The saddle points are the $k_{2}$ 's that satisfy the derivative of the exponent of the Fourier kernel,

$$
\frac{\partial}{\partial k_{2}}\left[i k_{1, j 0}\left[k_{2}\right] \cos \theta-\epsilon k_{1, j 1}\left[k_{2}\right] \cos \theta+i k_{2} \sin \theta\right]=0,
$$

for $j=1$ or 2. Equation (52) has two roots, namely $k_{2, j}^{ \pm}$for each $j$. We expand $k_{2}$ similarly to order $\epsilon$ to satisfy the above equation. Therefore, we write, for $j=1$ or 2 ,

$$
k_{2, j}^{ \pm} \approx k_{2, j 0}^{ \pm}[\theta]+i \epsilon k_{2, j 1}^{ \pm} .
$$

Upon substituting (53) into (52), it shows that

$$
k_{2, j 1}^{ \pm}[\theta]=-\left.\frac{\partial k_{1, j 1} / \partial k_{2}}{\partial^{2} k_{1, j 0} / \partial k_{2}^{2}}\right|_{k_{2}=k_{2, j 0}^{ \pm}} .
$$

We return to evaluate the integral expressions (43) and (44) at the saddle points. Here (43) and (44) can be written, in terms of saddle point contributions evaluated at $k_{2, j}^{ \pm}$, as ${ }^{12}$

$$
\begin{aligned}
\eta_{\mathrm{FS}}^{0} \approx \eta_{0}= & i F \sum_{j} \sqrt{\frac{2 \pi}{R\left|\partial^{2} k_{1, j} / \partial k_{2}\right| \cos \theta}} \\
\times & \operatorname{Res}\left[h_{0}\right] e^{i R\left[k_{1, j} \cos \theta+k_{2, j}^{ \pm} \sin \theta+(i / 2)\left(\pi-\delta_{j}\right)\right]}, \\
\eta_{\mathrm{FS}}^{v} \approx \epsilon^{1 / 2} \eta_{1}= & i F \sum_{j} \sqrt{\frac{2 \pi}{R\left|\partial^{2} k_{1, j} / \partial k_{2}\right| \cos \theta}} \\
& \times \operatorname{Res}\left[h_{1}\right] e^{i R\left[k_{1, j} \cos \theta+k_{2, j}^{ \pm} \sin \theta+(i / 2)\left(\pi-\delta_{j}\right)\right]},
\end{aligned}
$$

where the phase angle $\delta_{j}$ is defined through the relationship

$$
i \frac{\partial^{2} k_{1, j}}{\partial k_{2}}=\left|\frac{\partial^{2} k_{1, j}}{\partial k_{2}}\right| e^{i \delta_{j}} .
$$

Substituting (53) into the exponent of (55) and (56), we have

$$
\begin{aligned}
& i R\left(k_{1, j} \cos \theta+k_{2, j}^{ \pm} \sin \theta\right) \\
& \quad \approx i R\left[\left(k_{1, j 0}+i \epsilon k_{1, j 1}\right) \cos \theta+\left(k_{2, j 0}^{ \pm}+i \epsilon k_{2, j 1}^{ \pm}\right) \sin \theta\right] .
\end{aligned}
$$

From (54), it can be shown that $k_{2, j 1}^{ \pm}$is real, and thus

$$
\begin{aligned}
& i R\left(k_{1, j} \cos \theta+k_{2, j}^{ \pm} \sin \theta\right) \\
& \quad \approx-\epsilon R k_{1, j 1} \cos \theta+i R\left(k_{1, j 0} \cos \theta+k_{2, j 0}^{ \pm} \sin \theta\right)
\end{aligned}
$$

and

$$
\left|\frac{\partial k_{1, j 0}}{\partial k_{2}}\right|_{k_{2}=k_{2, j 0}^{ \pm}} \cos \theta+\sin \theta=0, \quad \text { for } j=1 \text { or } 2 .
$$

Returning to (57), we expand the denominator of the saddle point contributions about $\epsilon=0$, and keep only the terms of order $\epsilon$ or lower. From this and Eqs. (57)-(60), the phase angle in (57) can be found as

$$
\delta_{j}=\pi-\frac{\pi}{2} \operatorname{sgn}\left|\left(\frac{\partial^{2} k_{1, j 0}}{\partial k_{2}}\right)\right|_{k_{2}=k_{2, j 0}^{ \pm}}+O[\epsilon] .
$$

By substituting $k_{2, j 0}$ for $k_{2}$ into the residue function in (49) and (50), we obtain the leading term of it for small $\epsilon$, 


$$
\begin{aligned}
& \operatorname{Res}\left[h_{0}\right]=-i \frac{1+m\left[k_{2, j 0}^{ \pm}\right]}{2 m\left[k_{2, j 0}^{ \pm}\right]} e^{\left(z_{0} / 2\right)\left(1+m\left[k_{2, j 0}^{ \pm}\right]\right)}, \\
& \operatorname{Res}\left[h_{1}\right]=\epsilon^{1 / 2} \frac{2(-1)^{j-1}}{m\left[k_{2, j 0}^{ \pm}\right]}\left(\frac{1+m\left[k_{2, j 0}^{ \pm}\right]}{2}\right)^{7 / 4}
\end{aligned}
$$

$$
\times e^{-\left(z_{0} / \sqrt{2 \epsilon}\right)\left[\left(1+m\left[k_{2, j}^{ \pm}\right) / 2\right]\left[1+i(-1)^{j-1}\right]+i(-1)^{j-1}(\pi / 4)\right.} .
$$

Substituting the expansions into (57), we obtain the summation representation of the wave amplitude evaluated at $k_{2, j 0}^{ \pm}$,

$$
\begin{aligned}
\eta_{0}= & 2 F \sum_{ \pm} \sum_{j}\left(R\left|\frac{\partial^{2} k_{1, j 0}}{\partial k_{2}^{2}} \cos \theta\right|\right)^{-1 / 2} \frac{1+m^{ \pm}}{2 m^{ \pm}} e^{-\left(z_{0} / 2\right)\left(1+m^{ \pm}\right)-\epsilon R k_{1, j 1} \cos \theta+i R\left(k_{1, j 0} \cos \theta+k_{2, j 0}^{ \pm}\right)+i(\pi / 4) \operatorname{sgn}\left[\partial^{2} k_{1, j 0} / \partial k_{2}^{2}\right],} \\
\eta_{1}= & 4 i F \sum_{ \pm} \sum_{j}\left(R\left|\frac{\partial^{2} k_{1, j 0}}{\partial k_{2}^{2}} \cos \theta\right|\right)^{-1 / 2} \frac{(-1)^{j-1}}{m^{ \pm}}\left(\frac{1+m^{ \pm}}{2}\right)^{7 / 4} \\
& \times e^{-\left(z_{0} / \sqrt{2 \epsilon}\right)\left[\left(1+m^{ \pm}\right) / 2\right]^{1 / 4}\left[1+(-1)^{j-1}{ }_{i}-\epsilon R k_{1, j 1} \cos \theta+i R\left(k_{1, j 0} \cos \theta+k_{2, j 0}^{ \pm}\right)+i(\pi / 4) \operatorname{sgn}\left[\partial^{2} k_{1, j 0} / \partial k_{2}^{2}\right]\right]+i(-1)^{j-1}(\pi / 4)},
\end{aligned}
$$

where $\Sigma_{ \pm}$stands for the summation over the saddle points $k_{2, j}^{ \pm}$and

$$
m^{ \pm}=\sqrt{1+4\left(k_{2, j 0}^{ \pm}\right)^{2}} \text {. }
$$

The final procedure is to express all $k$ 's in terms of $\theta$. From Eqs. (45)-(48), we obtain

$$
k_{2, j 0}^{ \pm}=(-1)^{j} m^{ \pm}\left(\frac{1+m^{ \pm}}{2}\right)^{1 / 2} \tan \theta,
$$

with the root $m^{ \pm}$of this equation written as

$$
m^{ \pm}=\frac{1}{4} \cot ^{2} \theta\left(1 \pm \sqrt{1-8 \tan ^{2} \theta}\right) .
$$

Upon substituting and other mathematical manipulation, the wave amplitude can be formally expressed as

$$
\begin{aligned}
& \eta_{0}=4 F\left(\frac{2 \pi}{R \cos \theta}\right)^{1 / 2}\left(1-8 \tan ^{2} \theta\right)^{-1 / 4} \times\left\{\left(\frac{m^{+}+1}{2}\right)^{3 / 4} e^{-\left(z_{0} / 2\right)\left(1+m^{+}\right)-\left(4 \epsilon R / m^{+}\right)\left[\left(m^{+}+1\right) / 2\right]^{3} \cos \theta}\right. \\
& \times \cos \left[R\left(\frac{m^{+}+1}{2}\right)^{1 / 2}\left(\cos \theta-\left(\frac{m^{+}-1}{2}\right)^{1 / 2} \sin \theta\right)-\frac{\pi}{4}\right]+\left(\frac{m^{-}+1}{2}\right)^{3 / 4} e^{-\left(z_{0} / 2\right)\left(1+m^{-}\right)-\left(4 \epsilon R / m^{-}\right)\left[\left(m^{-}+1\right) / 2\right]^{3} \cos \theta} \\
& \left.\times \cos \left[R\left(\frac{m^{-}+1}{2}\right)^{1 / 2}\left(\cos \theta-\left(\frac{m^{-}-1}{2}\right)^{1 / 2} \sin \theta\right)+\frac{\pi}{4}\right]\right\} \\
& \eta_{1}=4 \sqrt{2} F\left(\frac{2 \pi}{R \cos \theta}\right)^{1 / 2}\left(1-8 \tan ^{2} \theta\right)^{-1 / 4} \times\left\{\left(\frac{m^{+}+1}{2}\right)^{3 / 2} e^{-\left(z_{0} / \sqrt{2 \epsilon}\right)\left(1+m^{+}\right)^{1 / 4}-\left(4 \epsilon R / m^{+}\right)\left[\left(m^{+}+1\right) / 2\right]^{3}} \cos \theta\right. \\
& \times \cos \left[R\left(\frac{m^{+}+1}{2}\right)^{1 / 2}\left(\cos \theta-\left(\frac{m^{+}-1}{2}\right)^{1 / 2} \sin \theta\right)-\frac{z_{0}}{\sqrt{2 \epsilon}}\left(1+m^{+}\right)^{1 / 4}+\frac{\pi}{2}\right] \\
& +\left(\frac{m^{-}+1}{2}\right)^{3 / 2} e^{-\left(z_{0} / \sqrt{2 \epsilon}\right)\left(1+m^{-}\right)^{1 / 4}-4 \epsilon R / m^{-}\left[\left(m^{-}+1\right) / 2\right]^{3} \cos \theta} \times \cos \left[R\left(\frac{m^{-}+1}{2}\right)^{1 / 2}\right. \\
& \left.\left.\times\left(\cos \theta-\left(\frac{m^{-}-1}{2}\right)^{1 / 2} \sin \theta\right)-\frac{z_{0}}{\sqrt{2 \epsilon}}\left(1+m^{-}\right)^{1 / 4}+\pi\right]\right\} .
\end{aligned}
$$

An immediate conclusion can be made about the wave amplitude regarding its region of validity. It can be seen that the expression breaks down as $\left(1-8 \tan ^{2} \theta\right) \geqslant 0$. This is just the same as in the classical inviscid Kelvin's wave pattern: surface deformation only occurs within a confined region of $|\theta| \leqslant \sin ^{-1}\left(\frac{1}{3}\right)$. Equations (69) and (70) break down even when $\theta=\sin ^{-1}\left(\frac{1}{3}\right)$, however, this can be taken care of by the extension of the method of steepest descent of uniform asymptotics of Chester, Friedman, and Ursell. ${ }^{12}$

Figures 1-3 show the normalized free-surface profiles generated by the horizontal Oseenlet for $\epsilon=0.01, z_{0}=1$, $F=\frac{1}{4}$, and $R>10$. Close examination of (69) and (70) and 


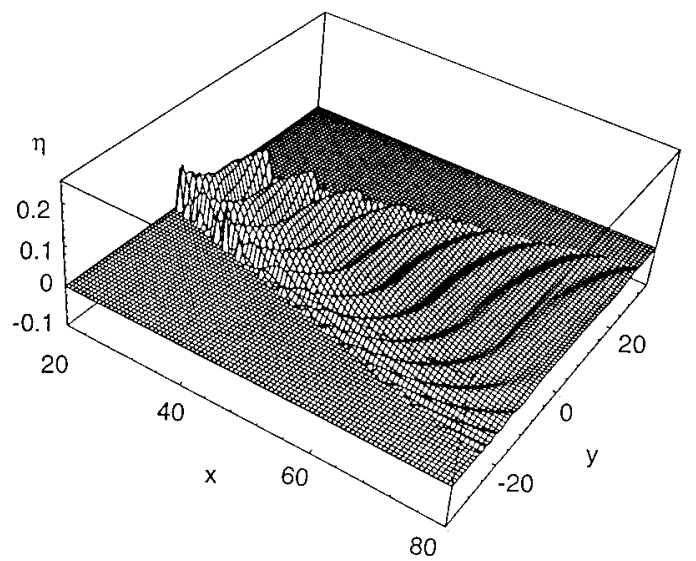

FIG. 1. Free-surface profile created by a horizontal Oseenlet.

Fig. 2 shows that the expressions involving $m^{+}$represent the diverging wave system, while those involving $m^{-}$represent the transverse system. From the two wave amplitude expressions, it can be seen that there exists a phase shift of $\pi / 2$ between them, which is the same as in the inviscid case. The wavelength of the diverging wave system, calculated from the phase equation of (69) and (70) is $4 \pi / \sqrt{ } 3$, and the angle of deviation subtended by the diverging and the transverse wave system with the line of motion at Kelvin's wedge is found to be $\frac{1}{2}\left[\pi / 2-\sin ^{-1}\left(\frac{1}{3}\right)\right]$, all being reconciled with the inviscid case.

In the limit as $\epsilon$ approaches zero, similar to the use of artificial viscosity in classical calculations of inviscid waves, Eq. (69) simply reduces to the classical Kelvin's wave pattern produced by a source of normalized strength $4 F$, as calculated by Wehausen and Laitone, ${ }^{4}$

$$
\begin{aligned}
\eta_{0}= & 4 F\left(\frac{2 \pi}{R \cos \theta}\right)^{1 / 2}\left(1-8 \tan ^{2} \theta\right)^{-1 / 4} \\
& \times\left\{( \frac { m ^ { + } + 1 } { 2 } ) ^ { 3 / 4 } e ^ { - ( z _ { 0 } / 2 ) ( 1 + m ^ { + } ) } \operatorname { c o s } \left[R\left(\frac{m^{+}+1}{2}\right)^{1 / 2}\right.\right. \\
& \left.\times\left(\cos \theta-\left(\frac{m^{+}-1}{2}\right)^{1 / 2} \sin \theta\right)-\frac{\pi}{4}\right] \\
& +\left(\frac{m^{-}+1}{2}\right)^{3 / 4} e^{-\left(z_{0} / 2\right)\left(1+m^{-}\right)} \cos \left[R\left(\frac{m^{-}+1}{2}\right)^{1 / 2}\right. \\
& \left.\left.\times\left(\cos \theta-\left(\frac{m^{-}-1}{2}\right)^{1 / 2} \sin \theta\right)+\frac{\pi}{4}\right]\right\}
\end{aligned}
$$

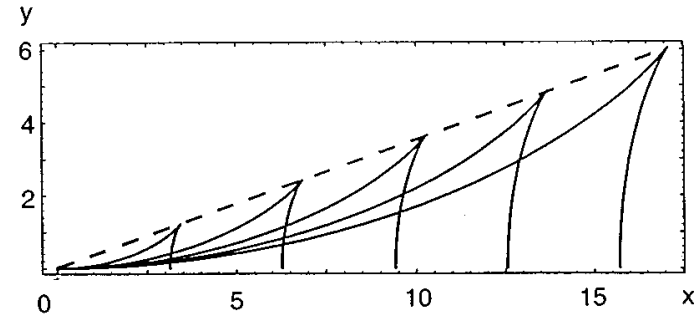

FIG. 2. Wave pattern generated by a horizontal Oseenlet/Oseen doublet.

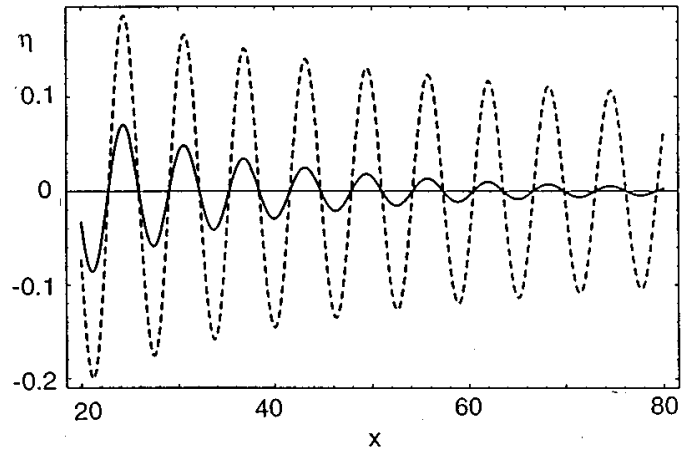

FIG. 3. Wave amplitude due to a horizontal Oseenlet versus the distance along the line of motion, and the dotted line represents the corresponding inviscid solution.

while Eq. (70) simply vanishes. The main difference between (69) and (71) is the presence of a viscous decay factor, namely

$$
e^{\left(4 \epsilon R / m^{ \pm}\right)\left[\left(1+m^{ \pm}\right) / 2\right]^{3} \cos \theta} .
$$

This viscous decay effect can be seen in Fig. 3 .

Since $\eta_{1}$ is associated with the $\epsilon^{1 / 2}$ term, as shown in (51), its contribution toward wave generation remains negligible unless the Oseenlet is very close to the free surface, that is, when the exponential terms $e^{-\left(z_{0} / \sqrt{2 \epsilon}\right)\left(1+m^{ \pm}\right)^{1 / 4}}$ in (70) become significant. From (70), as $\theta$ tends to zero, the diverging waves first rise to a sharp peak near the line of motion and then drop rapidly to 0 at $\theta=0$. This is in contrast with the inviscid theory, which predicts infinite diverging wave amplitude at $\theta=0$. Hence, it can be stated that the effect of viscosity is to remove this singularity.

\section{THEORETICAL SOLUTION FOR A HORIZONTAL OSEEN DOUBLET}

To simulate the flow over a self-propelling body, we investigate the case of an Oseen doublet. The analysis follows exactly that of the Oseenlet, with only minor modifications. Since the flow field over a doublet can be taken as the gradient of that of the source, therefore the velocity and pressure fields of a horizontal Oseen doublet with strength $4 \pi \Gamma$ are

$$
\begin{aligned}
& \mathbf{u}=\frac{\Gamma}{\epsilon} \frac{\partial}{\partial x} \frac{e^{(x-r) / 2 \epsilon}}{r} \mathbf{e}_{x}-\Gamma \frac{\partial}{\partial x} \nabla\left[\frac{e^{(x-r) / 2 \epsilon}-1}{r}\right], \\
& p=\frac{\partial}{\partial x} \frac{\Gamma x}{r^{3}}
\end{aligned}
$$

where

$$
\Gamma=F d x
$$

Using similar procedures and defining the Fourier transform,

$$
\begin{aligned}
& \phi=\Gamma \int_{-\infty}^{\infty} \int_{-\infty}^{\infty} \hat{\phi}\left[k_{1}, k_{2}\right] e^{i\left(k_{1} x+k_{2} y\right)+A z} d k_{1} d k_{2}, \\
& f_{j}=\Gamma \int_{-\infty}^{\infty} \int_{-\infty}^{\infty} \hat{f}_{j}\left[k_{1}, k_{2}\right] e^{i\left(k_{1} x+k_{2} y\right)+B z} d k_{1} d k_{2},
\end{aligned}
$$

we obtain, after some mathematical manipulation, 
$\hat{\phi}_{0}=-i k_{1} \frac{\left[-\left(k_{1}^{2} / 2 A+\frac{1}{2}\right)+2 i \epsilon k_{1} A+2 \epsilon^{2} A^{2}(A+B)\right] e^{-A z_{0}}}{\Delta}$,

$\hat{\phi}_{v}=-i k_{1} \frac{\left(-2 i \epsilon k_{1} B-4 \epsilon^{2} A^{3} B\right) e^{-B z_{0}}}{\Delta}$,

$\hat{f}_{0}=-i k_{1} \frac{\left(-2 \epsilon k_{1} A^{2}+4 i \epsilon^{2} A^{4}\right) e^{-A z_{0}}}{k_{2} \Delta}$,

$\hat{f}_{v}=-i k_{1}$

$$
\times \frac{\left[(i / 2)\left(k_{1}^{2}-A\right)+2 \epsilon k_{1} A^{2}-2 i \epsilon^{2} A^{4}-2 i \epsilon^{2} A^{3} B\right] e^{-B z_{0}}}{k_{2} \Delta},
$$

where $\Delta$ is given in (37). The integral form of the surface profile can then be expressed as

$$
\eta_{\mathrm{FS}}^{0}=\Gamma \int_{-\infty}^{\infty} \int_{-\infty}^{\infty} h_{0} e^{i R\left(k_{1} \cos \theta+k_{2} \sin \theta\right)} d k_{1} d k_{2},
$$

$$
\begin{aligned}
\eta_{0}= & -2 \Gamma\left(\frac{2 \pi}{R \cos \theta}\right)^{1 / 2}\left(1-8 \tan ^{2} \theta\right)^{-1 / 4}\left\{\left(\frac{m^{+}+1}{2}\right)^{5 / 4} e^{-\left(z_{0} / 2\right)\left(1+m^{+}\right)-\left(4 \epsilon R / m^{+}\right)\left[\left(m^{+}+1\right) / 2\right] \cos \theta}\right. \\
& \times \cos \left[R\left(\frac{m^{+}+1}{2}\right)^{1 / 2}\left(\cos \theta-\left(\frac{m^{+}-1}{2}\right)^{1 / 2} \sin \theta\right)+\frac{\pi}{4}\right]+\left(\frac{m^{-}+1}{2}\right)^{5 / 4} e^{-\left(z_{0} / 2\right)\left(1+m^{-}\right)-\left(4 \epsilon R / m^{-}\right)\left(m^{-}+1\right) / 2 \cos \theta} \\
& \left.\times \cos \left[R\left(\frac{m^{-}+1}{2}\right)^{1 / 2}\left(\cos \theta-\left(\frac{m^{-}-1}{2}\right)^{1 / 2} \sin \theta\right)+\frac{3 \pi}{4}\right]\right\}, \\
\eta_{1}= & -2 \sqrt{2} \Gamma\left(\frac{2 \pi}{R \cos \theta}\right)^{1 / 2}\left(1-8 \tan ^{2} \theta\right)^{-1 / 4}\left\{\left(\frac{m^{+}+1}{2}\right)^{2} e^{-\left(z_{0} / \sqrt{2 \epsilon}\right)\left(1+m^{+}\right)^{1 / 4}-\left(4 \epsilon R / m^{+}\right)\left[\left(m^{+}+1\right) / 2\right]^{3} \cos \theta} f\right. \\
& \times \cos \left[R\left(\frac{m^{+}+1}{2}\right)^{1 / 2}\left(\cos \theta-\left(\frac{m^{+}-1}{2}\right)^{1 / 2} \sin \theta\right)-\frac{z_{0}}{\sqrt{2 \epsilon}}\left(1+m^{+}\right)^{1 / 4}\right] \\
& +\left(\frac{m^{-}+1}{2}\right)^{2} e^{-\left(z_{0} / \sqrt{2 \epsilon}\right)\left(1+m^{-}\right)^{1 / 4}-\left(4 \epsilon R / m^{-}\right)\left[\left(m^{-}+1\right) / 2\right]^{3} \cos \theta} \\
& \left.\times \cos \left[R\left(\frac{m^{-}+1}{2}\right)^{1 / 2}\left(\cos \theta-\left(\frac{m^{-}-1}{2}\right)^{1 / 2} \sin \theta\right)-\frac{z_{0}}{\sqrt{2 \epsilon}}\left(1+m^{-}\right)^{1 / 4}+\frac{\pi}{2}\right]\right\} .
\end{aligned}
$$

Figures 4 and 5 give the graphical plots of a horizontal Oseen doublet with $\epsilon=0.01, z_{0}=1, \Gamma=\frac{1}{4}$, and large $R$. The wave pattern due to an Oseen doublet is very similar to that produced by an Oseenlet, except that the former lags the latter by a phase of $\pi / 2$. The wave amplitude generated is seen to be much reduced because of the decoupling effect of the doublet.

\section{THEORETICAL SOLUTION FOR A VERTICAL OSEENLET}

We now investigate the surface waves generated by an Oseenlet moving perpendicularly away from the free surface with velocity $U$. For the wake to interact with the free surface, the Oseenlet must move away from it. Since the flow is basically potential upstream and outside of the laminar wake 
$\eta$

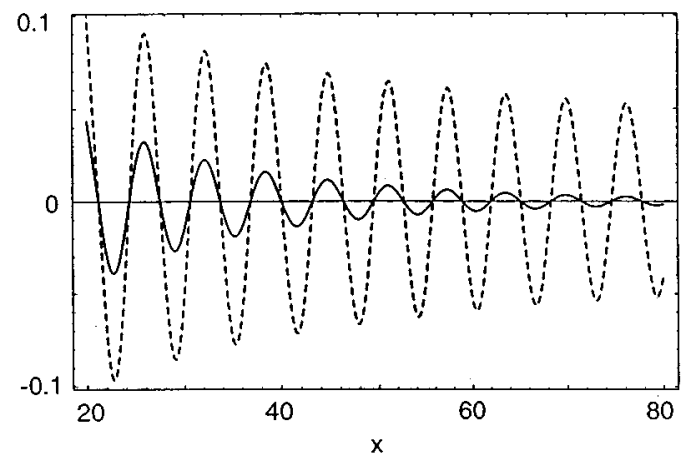

FIG. 5. Wave amplitude due to a horizontal Oseen doublet versus distance along the line of motion and the dotted line represents the corresponding inviscid solution.

of the singularity, the free-surface deformation caused by the Oseenlet moving toward the free surface is negligible when compared with that generated by the wake. Therefore we shall concentrate on investigating the effect of an Oseenlet moving away from the free surface only. Assuming sufficiently large depth so that the Oseen equations can be employed, the solution to (1) and (2) for a singular force located at $\left(0,0,-z_{0}\right)$ with a magnitude $4 \pi F_{z}$ along the negative $z$ direction in an unbounded fluid is then

$$
\begin{aligned}
\mathbf{u}_{u} & =-\frac{F_{z}}{\epsilon} \frac{e^{\left(z+z_{0}-r\right) / 2 \epsilon}}{r} \mathbf{e}_{z}+F_{z} \boldsymbol{\nabla}\left[\frac{e^{\left(z+z_{0}-r\right) / 2 \epsilon}-1}{r}\right], \\
p_{u} & =-\frac{F_{z}\left(z+z_{0}\right)}{r^{3}} .
\end{aligned}
$$

We shall employ cylindrical coordinates $(R, \theta, z)$ to deal with this axisymmetric problem. The free-surface boundary conditions are given by (7)-(9), except that (9) is replaced by

$$
\frac{\partial p}{\partial z}-2 \epsilon \frac{\partial^{2} w}{\partial z^{2}}-w=0
$$

for $z=0$, where $\mathbf{u}=(u, v, w)$ is the normalized velocity vector in the polar coordinate system.

We introduce similarly the free-surface potential and streamfunctions such that

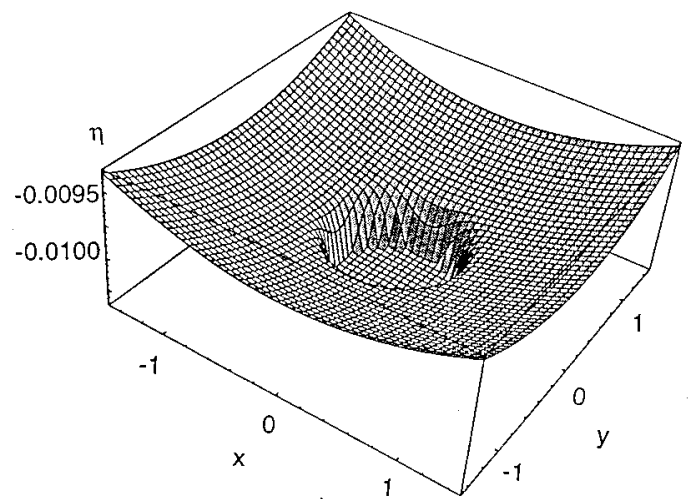

FIG. 6. Free surface profile generated by a vertical Oseenlet.

$$
\mathbf{u}_{\mathrm{FS}}=-\boldsymbol{\nabla} \phi+\nabla \times \mathbf{f},
$$

with

$$
\mathbf{f}=f_{z} \mathbf{e}_{z}+f_{R} \mathbf{e}_{R} .
$$

After some mathematical manipulation, the free-surface conditions can be expressed in terms of $\phi$ only as

$$
\frac{\partial^{2} \phi}{\partial z^{2}}-2 \epsilon \frac{\partial^{3} \phi}{\partial z^{3}}-\frac{\partial \phi}{\partial z}=-\left(\frac{\partial p_{u}}{\partial z}-2 \epsilon \frac{\partial^{2} w_{u}}{\partial z^{2}}-w_{u}\right) .
$$

Since $\phi$ satisfies the cylindrical Laplacian, we introduce a Hankel transform defined by

$$
\phi=F_{z} \int_{0}^{\infty} \hat{\phi}[k] e^{-k z} J_{0}[k R] d k,
$$

where $J_{0}[x]$ is the Bessel function of the zeroth order.

Recalling the expression for the wave amplitude,

$$
\eta=\left.p\right|_{z=0}-\left.2 \epsilon \frac{\partial w}{\partial z}\right|_{z=0}=\eta_{u}+\eta_{\mathrm{FS}}
$$

it must be emphasized that $\eta_{u}$ is not negligible in this case. Employing similar techniques, the free-surface potential functions $\hat{\phi}$ can be expressed algebraically as

$$
\hat{\phi}_{0}=-e^{-k z_{0}},
$$

$$
\hat{\phi}_{v}=\frac{\left(a^{2}+a\right)+(1-a) \sqrt{k^{2}+a^{2}}+(2+3 a)\left(k^{2}+a^{2}\right)-\left(k^{2}+a^{2}\right)^{3 / 2}}{\sqrt{k^{2}+a^{2}}\left(\epsilon k^{2}-k+1\right)} e^{z_{0}\left(a-\sqrt{k^{2}+a^{2}}\right)},
$$

where subscripts 0 and $v$ have the same meaning as in the previous section and

$$
a=\frac{1}{2 \epsilon} \text {. }
$$

Hence, the exact integral form of the wave amplitude can be formulated as

$$
\begin{aligned}
& \eta_{0}=F_{z} \int_{0}^{\infty} h_{0} J_{0}[k R] d k, \\
& \eta_{v}=F_{z} \int_{0}^{\infty} h_{v} J_{0}[k R] d k,
\end{aligned}
$$

where 


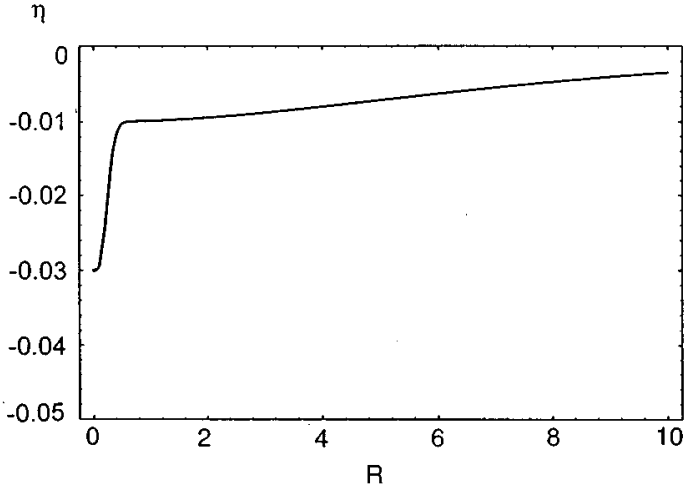

FIG. 7. Wave amplitude of a vertical Oseenlet versus the radial distance.

$$
\begin{aligned}
& h_{0}=\left(-k+2 \epsilon k^{2}\right) \hat{\phi}_{0}, \\
& h_{v}=\left(-k+2 \epsilon k^{2}\right) \hat{\phi}_{v},
\end{aligned}
$$

From the theory of the Hankel transform, ${ }^{14}$ it can be seen that the first integral is directly integrable, giving rise to an exact solution,

$$
\begin{aligned}
\eta_{0}= & F_{z}\left(-\frac{{ }_{2} F_{1}\left[1,-\frac{1}{2} ; 1 ; R^{2} /\left(R^{2}+z_{0}^{2}\right)\right]}{\sqrt{R^{2}+z_{0}^{2}}}\right. \\
& \left.+\frac{4 \epsilon_{2} F_{1}\left[3,-1 ; 1 ; R^{2} /\left(R^{2}+z_{0}^{2}\right)\right]}{\left(R^{2}+z_{0}^{2}\right)^{3 / 2}}\right) e^{-z_{0} / \epsilon},
\end{aligned}
$$

where ${ }_{2} F_{1}[a, b ; c ; z]$ is the 2-1 hypergeometric function. On the other hand, the $\eta_{v}$ term is simply a series of Cauchy Principal Values. However, by Laplace's asymptotic method, it can be found that all of them are of the order of $\epsilon e^{-z_{0} / \epsilon}$ or lower, and thus can be neglected for large values of $z_{0}$. Thus, the first two terms of the wave amplitude expansion following (51) are

$$
\begin{aligned}
& \eta_{0}=F_{z}\left(-\frac{{ }_{2} F_{1}\left[1,-\frac{1}{2} ; 1 ; R^{2} /\left(R^{2}+z_{0}^{2}\right)\right]}{\sqrt{R^{2}+z_{0}^{2}}}\right) e^{-z_{0} / \epsilon}, \\
& \eta_{1}=0 .
\end{aligned}
$$

The normalized graphical plots of the vertical Oseenlets with $\epsilon=0.01, z_{0}=10$, and $F_{z}=1$ are presented in Fig. 6 and Fig. 7. Based on the expressions and the figures, it can be seen that the decay of the ripples is much quickened by the

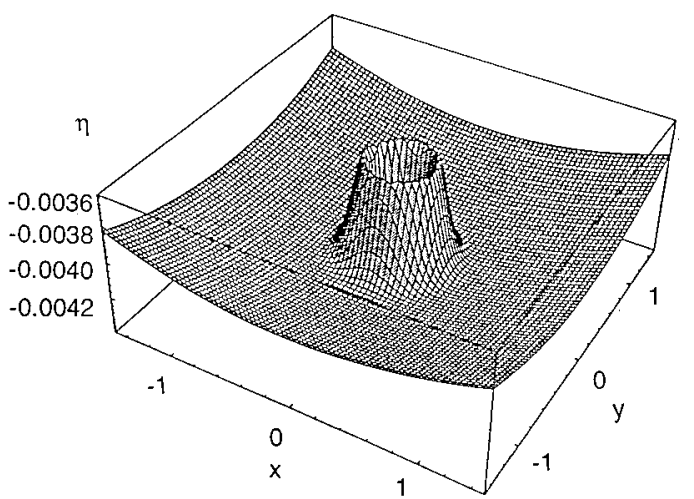

FIG. 8. Free-surface profile generated by a vertical Oseen doublet.

effect of viscosity. In the inviscid case, the ripples decay exponentially. It is observed that the wave amplitude from the free-surface effect term $\eta_{\mathrm{FS}}$ is negligible when compared to the unbounded solution $\eta_{u}$. This is in large contrast with the horizontal Oseenlet, where the main contribution arises from the free-surface solution, as seen from Eq. (30). This can be explained from the fact that as the Oseen equations are employed, large submergence must be assumed. As the free-surface solution decays like $\exp \left[-z_{0} / \epsilon\right]$, the solution becomes negligible.

\section{THEORETICAL SOLUTION FOR A VERTICAL OSEEN DOUBLET}

We continue to consider the effect of a self-propelling body moving vertically downward away from the free surface. The velocity and pressure fields of a vertical Oseen doublet with strength $4 \pi \Gamma_{z}$ can be expressed as

$$
\begin{aligned}
& \mathbf{u}_{u}=\frac{\Gamma_{z}}{\epsilon} \frac{\partial}{\partial z} \frac{e^{\left(z+z_{0}-r\right) / 2 \epsilon}}{r} \mathbf{e}_{z}-\Gamma_{z} \frac{\partial}{\partial z} \nabla\left[\frac{e^{\left(z+z_{0}-r\right) / 2 \epsilon}-1}{r}\right], \\
& p_{u}=\frac{\partial}{\partial z} \frac{\Gamma_{z}\left(z+z_{0}\right)}{r^{3}},
\end{aligned}
$$

where

$$
\Gamma_{z}=F_{z} d z
$$

Using exactly the same procedure, but replacing all $F_{z}$ by $\Gamma_{z}$, we can obtain the free-surface potential functions as

$$
\hat{\phi}_{0}=k e^{-k z_{0}},
$$

$$
\hat{\phi}_{v}=-\frac{\left(a^{2}+a\right)+(1-a) \sqrt{k^{2}+a^{2}}+(2+3 a)\left(k^{2}+a^{2}\right)-\left(k^{2}+a^{2}\right)^{3 / 2}}{\sqrt{k^{2}+a^{2}}\left(\epsilon k^{2}-k+1\right)}\left(a-\sqrt{k^{2}+a^{2}}\right) e^{z_{0}\left(a-\sqrt{k^{2}+a^{2}}\right)},
$$


$\eta$

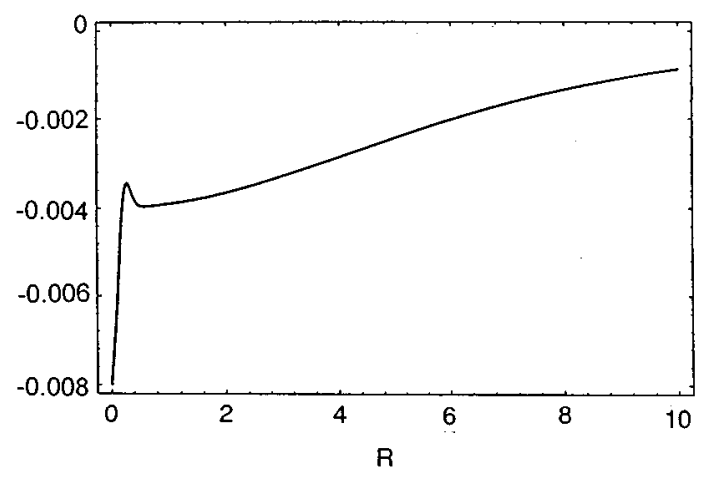

FIG. 9. Wave amplitude of a vertical Oseen doublet versus the radial distance.

where $a$ is given in (95). Again, an exact solution for the wave amplitude by the inviscid term can be obtained in terms of

$$
\eta_{0}=-\Gamma_{z}\left(-\frac{z_{0}}{\left(R^{2}+z_{0}^{2}\right)^{3 / 2}}-\frac{2 \epsilon z_{0}}{\left(R^{2}+z_{0}^{2}\right)^{5 / 2}}\right) e^{-z_{0} / \epsilon},
$$

and by Bleistein asymptotic evaluation the viscous term turns out to be of the order of $\epsilon \exp \left[-z_{0} / \epsilon\right]$ and is thus neglected. Thus, the first two terms of the wave amplitude expansion of the free-surface contribution can be expressed as

$$
\begin{aligned}
& \eta_{0}=\Gamma_{z} \frac{z_{0}}{\left(R^{2}+z_{0}^{2}\right)^{3 / 2}} e^{-z_{0} / \epsilon}, \\
& \eta_{1}=0 .
\end{aligned}
$$

The graphical plots of a vertical Oseen doublet are presented in Fig. 8 and Fig. 9 for $\epsilon=0.01, z_{0}=10$, and $\Gamma_{z}=1$. It can be seen that the wave amplitude is less conspicuous than that of a vertical Oseenlet. Besides, the waves also decay much faster than the former case.

\section{CONCLUSIONS}

With the Oseen flow assumption, the asymptotic expressions and graphical representations of free-surface profiles caused by the translatory motion of an object are presented.
Though extensive work on surface waves has been known, the inclusion of the laminar wake is believed to be new. The graphical plots of the free-surface profiles are in fairly good agreement with physical intuition. However, it must be emphasized that the use of the linearized theory can only predict the leading-order solutions for the actual problem.

The present work is a first attempt. Possible cases such as wave generation in waters of finite depth, transient development of viscous waves may also be of interest in engineering and physical applications. The use of linearized theory has also shed light on the hypothetical possibility of superposition to simulate other types of flow over a finite body. All of the aforementioned work will require extensive investigation and will be discussed in future studies.

\section{ACKNOWLEDGMENTS}

The authors are indebted to Dr. G. T. Yates for his valuable suggestions and comments. This work was sponsored by the Committee on Research and Conference Grants, The University of Hong Kong, under Grant No. 335/064/0071.

${ }^{1}$ W. Thomson, “Deep water ship waves," Mathematical and Physical Papers 4, pp. 303-306, 1910.

${ }^{2}$ H. Lamb, Hydrodynamics (Dover, New York, 1932).

${ }^{3}$ C. R. De Prima and T. Y. Wu, "On the theory of generating surface waves on water by moving obstacles," Proceedings of the 9th International Congress of Applied Mechanics, 1956, pp. 103-126.

${ }^{4}$ J. V. Wehausen and E. V. Laitone "Surface waves," Handbuch der Physik 8/3 Strömungsmechanik III (Springer-Verlag, New York, 1960), pp. 311778.

${ }^{5}$ E. Cumberbatch, "Effect of viscosity on ship waves," J. Fluid Mech. 23 471 (1965).

${ }^{6}$ J. P. Dugan, "Viscous drag of bodies moving near a free surface," Phys. Fluids 12, 1 (1969).

${ }^{7}$ E. Ammicht "Free surface and surface tension effects on submerged bodies," J. Eng. Math. 13, 327 (1979).

${ }^{8}$ J. R. Lurye, "Interaction of free surface waves with viscous wakes," Phys. Fluids 10, 261 (1968).

${ }^{9}$ F. K. Moore, Theory of Laminar Flows (Princeton University Press, Princeton, 1964).

${ }^{10}$ M. J. Lighthill "Studies on magneto-hydrodynamic waves and other anisotropic wave motions," Philos. Trans. R. Soc. London Ser. A 252, 397 (1960).

${ }^{11}$ J. Kevorkian and J. D. Cole, Perturbation Methods in Applied Mathematics (Springer-Verlag, New York, 1981).

${ }^{12} \mathrm{R}$. Wong, Asymptotic Approximations of Integrals (Academic, New York, 1989).

${ }^{13}$ C. Chester, B. Friedman, and F. Ursell, "An extension of the method of steepest descents," Proc. Cambridge Philos. Soc. 53599 (1957).

${ }^{14}$ G. N. Watson A Treatise on the Theory of Bessel Functions (Cambridge University Press, Cambridge, 1962). 